\title{
Association between intrinsic disorder and serine/threonine phosphorylation in Mycobacterium tuberculosis
}

Gajinder P Singh

Serine/threonine phosphorylation is an important mechanism that is involved in the regulation of protein function. In eukaryotes, phosphorylation occurs predominantly in intrinsically disordered regions of proteins. Though serine/threonine phosphorylation and protein disorder are much less prevalent in prokaryotes, some bacteria have high levels of serine/threonine phosphorylation and disorder, including the medically important $M$. tuberculosis. Here, I show that, serine/threonine phosphorylation sites in M. tuberculosis are highly enriched in intrinsically disordered regions, indicating similarity in the substrate recognition mechanisms of eukaryotic and $M$. tuberculosis kinases. Serine/threonine phosphorylation has been linked to the pathogenicity and survival of $M$. tuberculosis. Thus, a better understanding of how its kinases recognize their substrates could have important implications in understanding and controlling the biology of this deadly pathogen. These results also indicate that the association between serine/threonine and disorder is not a feature restricted to eukaryotes. 
1 Association between intrinsic disorder and serine/threonine phosphorylation in

2 Mycobacterium tuberculosis

3 Gajinder Pal Singh

4 School of Biotechnology,

5 KIIT University, Campus-XI, Patia, Bhubaneswar 751024,

6 Odisha, India

$7 \quad$ Tel : +91 6742725732

8 Email: gajinder.pal.singh@gmail.com

9 Abstract 
10 Serine/threonine phosphorylation is an important mechanism that is involved in the regulation of

11 protein function. In eukaryotes, phosphorylation occurs predominantly in intrinsically disordered

12 regions of proteins. Though serine/threonine phosphorylation and protein disorder are much less

13 prevalent in prokaryotes, some bacteria have high levels of serine/threonine phosphorylation and

14 disorder, including the medically important M. tuberculosis. Here, I show that, serine/threonine

15 phosphorylation sites in M. tuberculosis are highly enriched in intrinsically disordered regions,

16 indicating similarity in the substrate recognition mechanisms of eukaryotic and M. tuberculosis

17 kinases. Serine/threonine phosphorylation has been linked to the pathogenicity and survival of $M$.

18 tuberculosis. Thus, a better understanding of how its kinases recognize their substrates could have

19 important implications in understanding and controlling the biology of this deadly pathogen.

20 These results also indicate that the association between serine/threonine and disorder is not a

21 feature restricted to eukaryotes.

\section{Introduction}

23 The reversible phosphorylation of serine and threonine residues is a widespread post-translational

24 modification in eukaryotes, with more than a third of proteins phosphorylated during their

25 lifetime (Albuquerque et al., 2008). Phosphorylation can modify protein interactions, enzyme

26 functions, localization and degradation. Although regulation and signal transduction in bacteria

27 were traditionally thought to be mediated by histidine and aspartate phosphorylation in two-

28 component systems, the occurrence and importance of phosphorylation of serine/threonine ( $\mathrm{S} / \mathrm{T})$

29 residues has recently gained much attention (Cousin et al., 2013; Kobir et al., 2011; Mijakovic

30 and Macek, 2012). Large-scale mass spectrometry based analyses have revealed S/T

31 phosphorylation in a number of bacteria (Mijakovic and Macek, 2012). 
32 One of the most interesting findings concerning $\mathrm{S} / \mathrm{T}$ phosphorylation in eukaryotes is its

33 association with intrinsically disordered regions (Amoutzias et al., 2012; Collins et al., 2008;

34 Gnad et al., 2009; Gsponer et al., 2008; Iakoucheva et al., 2004; Marchini et al., 2011).

35 Intrinsically disordered regions lack a well-defined three-dimensional structure and are

36 characterized by low hydrophobic amino acid content and a high net charge (Uversky et al.,

37 2000). These characteristic physiochemical properties allow for accurate predictions of

38 disordered regions across proteomes (Monastyrskyy et al., 2014). Disordered regions are often

39 associated with the ability to bind to multiple partners in a transient manner (Dunker et al., 2005;

40 Fink, 2005; Patil and Nakamura, 2006; Singh et al., 2007; Tompa et al., 2005; Wright and Dyson,

41 1999). These regions may undergo a disorder to order transition upon binding, with decrease in

42 conformational entropy. This process uncouples binding affinity and specificity, thus allowing

43 highly specific interactions to be reversible (Dyson and Wright, 2005; Tompa, 2002). It has been

44 proposed that disordered regions are ideally suited for regulation by reversible phosphorylation

45 due to their high surface accessibility and transient mode of interactions (Collins et al., 2008;

46 Dyson and Wright, 2005; Iakoucheva et al., 2004; Tompa, 2002). Disordered regions are highly

47 abundant in eukaryotes, with approximately one third of proteins predicted to have at least one

48 long (> 30 residues) disordered region and approximately $19 \%$ of residues predicted to be in a

49 disordered state (Ward et al., 2004). In contrast, most bacteria have much less disorder in their

50 proteome, with approximately $4 \%$ proteins predicted to contain long disordered regions and

51 approximately $6 \%$ of residues predicted to be disordered (Ward et al., 2004). The association

52 between disorder and $\mathrm{S} / \mathrm{T}$ phosphorylation has not been investigated in prokaryotes.

53 Among bacteria, S/T phosphorylation is most well studied in M. tuberculosis and is linked to its

54 survival, pathogenesis and virulence (Av-Gay and Everett, 2000; Cousin et al., 2013; Pereira et 
55 al., 2011). This bacterium shows one of the highest rates of phosphorylation among studied

56 bacteria, with $8 \%$ of its proteins identified as phosphorylated (Prisic et al., 2010). M. tuberculosis

57 also has a high disorder content, with approximately 10\% disordered residues (Ward et al., 2004).

58 The prevalence and importance of $\mathrm{S} / \mathrm{T}$ phosphorylation in M. tuberculosis prompted the question

59 of whether the association observed between $\mathrm{S} / \mathrm{T}$ phosphorylation and disorder in eukaryotes

60 might be present in M. tuberculosis, which indeed was found to be the case. Furthermore, this

61 association was also identified in other bacteria.

\section{Materials and Methods}

63 Data on M. tuberculosis phosphoproteins and phosphosites were obtained from Prisic et al.

64 (Prisic et al., 2010). This study identified 301 phosphoproteins and $500 \mathrm{~S} / \mathrm{T}$ phosphorylation

65 events. For 215 of these sites, the specific residue that was modified could be identified with high

66 confidence. The M. tuberculosis proteome was obtained from the Tuberculist database (Lew et

67 al., 2013). For disorder prediction, I utilized the IUPred method (Dosztanyi et al., 2005a). This

68 method is based on the observation that disordered regions do not form sufficiently favorable

69 interactions to fold and thus have high estimated energy content (Dosztanyi et al., 2005b). I also

70 utilized the ESpritz program (Walsh et al., 2012), which is conceptually different from IUPred.

71 This method is a machine-learning based predictor that was trained on experimentally

72 characterized disordered regions (missing regions in X-ray structures in PDB). I also used the

73 MFDp2 disorder prediction tool, which is an ensemble disorder prediction tool (Mizianty MJ et

74 al., 2013; Mizianty et al., 2014). Secondary structure prediction was performed at the Network

75 Protein Sequence Analysis (NPSA) server (Combet et al., 2000) using a consensus approach

76 (Deleage et al., 1997). To analyze conservation of S/T sites, 14 diverse mycobacterium species

77 were chosen (M. intracellulare, M. smegmatis, M. chubuense, M. avium, M. gilvum, M.

78 abscessus, M. marinum, M. bovis, M. canettii, M. kansasi, Mycobacterium sp. MCS, 
79 Mycobacterium sp. JLS, Mycobacterium sp. KMS, and Mycobacterium sp. JDM60). Orthologs of

80 M. tuberculosis in mycobacteria were identified using the reciprocal best blast approach (Wolf

81 and Koonin, 2012), and aligned using Clustal Omega (Sievers and Higgins, 2014). Alignment

82 positions with gaps were excluded from the analyses. Of the 215 sites, 139 sites were present in

83 proteins which had orthologs in all other 14 species. Of these 139 sites, 103 sites were without

84 gaps. Positions with replacement of serine with threonine and vice-versa were considered to be

85 conserved. The number of species in which $\mathrm{S} / \mathrm{T}$ residues were present at the alignment position

86 was calculated as a measurement of conservation. Phosphosite data for other bacteria were

87 obtained from respective publications (Aivaliotis et al., 2009; Lin et al., 2009; Macek et al., 2007;

88 Manteca et al., 2011; Misra et al., 2011; Parker et al., 2010; Soufi et al., 2008; Yang et al., 2013).

\section{Results}

90 Mass-spectrometry based analysis previously revealed 301 phosphoproteins in M. tuberculosis

91 containing $500 \mathrm{~S} / \mathrm{T}$ phosphorylation sites (Prisic et al., 2010). First, I tested whether

92 phosphoproteins in M. tuberculosis were more likely to be disordered (i.e., have long ( $>=30$

93 residues) disordered regions). I utilized the IUPred program to predict disordered regions at the

94 proteome wide level (Dosztanyi et al., 2005b). Phosphoproteins were approximately twice as

95 likely to be disordered compared to non-phosphoproteins ( $29.6 \%$ vs. $13.4 \%$, respectively; Fisher

96 test $p$ 4e-12). Because longer proteins are also more likely to have long disordered regions, I

97 tested whether phosphoproteins have higher percentage of disordered residues. Indeed,

98 phosphoproteins have higher percentage of disordered residues than do non-phosphoproteins

$99(16.7 \%$ vs. $12.0 \%$, respectively; two tailed t-test $p 3 \mathrm{e}-5)$.

100 Of the 500 phosphorylation events detected in M. tuberculosis, the phosphoresidues could be

101 identified for 215 sites with high confidence (Prisic et al., 2010). For these sites, I tested whether 
102 phosphorylated $\mathrm{S} / \mathrm{T}(\mathrm{pS} / \mathrm{T})$ residues were more likely to be disordered compared to non-

103 phosphorylated $\mathrm{S} / \mathrm{T}(\mathrm{npS} / \mathrm{T})$ residues from the same set of proteins. Overall, $39.1 \%$ of the $\mathrm{pS} / \mathrm{T}$

104 sites were disordered compared with $22.4 \%$ of npS/T sites (Fisher test $p$ 6e-8; Figure 1). The

105 results were very similar when another disorder prediction method, ESpritz (Walsh et al., 2012),

106 was used (52.6\% pS/T disordered compared with $27.8 \% \mathrm{npS} / \mathrm{T}$ sites; Fisher test $p 8 \mathrm{e}-14$, Figure

107 1). The more recently described disorder predictor MFDp2 (Mizianty MJ et al., 2013; Mizianty et

108 al., 2014) also gave similar results (43.7\% pS/T disordered compared with 19.9\% npS/T sites;

109 Fisher test $p$ 6e-15; Figure 1). Disordered regions are also characterized by high irregular

110 secondary structure regions (i.e., coil regions). Thus, I tested whether $\mathrm{pS} / \mathrm{T}$ residues were

111 enriched in coil regions of the proteins. $\mathrm{pS} / \mathrm{T}$ residues were more likely to occur in predicted coil

112 regions than were $\mathrm{npS} / \mathrm{T}$ residues $(70.2 \% \mathrm{pS} / \mathrm{T}$ sites in coils compared with $55.7 \% \mathrm{npS} / \mathrm{T}$ sites in

113 coils; Fisher test $p$ 2e-5; Figure 2). A depletion of $\mathrm{pS} / \mathrm{T}$ residues in beta-sheet regions was also

114 observed (4.2\% pS/T sites in sheet compared with $11.3 \% \mathrm{npS} / \mathrm{T}$ sites in sheets; Fisher test $p$ 4e-4;

115 Figure 2), whereas no significant difference was found for helix regions (22.8\% $\mathrm{pS} / \mathrm{T}$ sites in

116 helices compared with $27.1 \% \mathrm{npS} / \mathrm{T}$ sites in helices; Fisher test $p$ 0.2; Figure 2).

117 Next, I tested whether M. tuberculosis $\mathrm{pS} / \mathrm{T}$ and $\mathrm{npS} / \mathrm{T}$ differed in the conservation across

118 mycobacteria. I identified orthologs of M. tuberculosis among 14 mycobacterial species, aligned

119 their sequences and calculated the ratio of conservation of $\mathrm{pS} / \mathrm{T}$ sites with $\mathrm{npS} / \mathrm{T}$ sites for each

120 phosphoprotein (see methods). In 65\% (49/76) of the proteins, $\mathrm{pS} / \mathrm{T}$ sites were more conserved

121 than $\mathrm{npS} / \mathrm{T}$ sites from the same protein (Supplemental Figure 1). This proportion was

122 significantly different from the expected value of $50 \%$ (Binomial test $p 0.008$ ). Because

123 disordered regions and disordered pS/T sites are known to evolve faster (Brown et al., 2002;

124 Landry et al., 2009), I analyzed the disordered and ordered sites separately. In 69\% (40/58) of the

125 proteins, ordered $\mathrm{pS} / \mathrm{T}$ sites were more conserved than ordered $\mathrm{npS} / \mathrm{T}$ sites (Binomial test $p$ 
$1260.003)$, whereas in $61 \%(14 / 23)$ of the proteins, disordered $\mathrm{pS} / \mathrm{T}$ sites were more conserved than

127 disordered $\mathrm{npS} / \mathrm{T}$ sites (Binomial test $p$ 0.2). It is likely that the lack of higher conservation of

128 disordered $\mathrm{pS} / \mathrm{T}$ sites might be due to the low number of sites and proteins analyzed.

129 Prisic et al. (Prisic et al., 2010) conducted in vitro phosphorylation of 13-mer synthetic peptides

130 corresponding to in vivo phosphorylation sites using different purified kinases. They could find

131 phosphorylation of approximately half of these peptides. Based on these results, I tested whether

132 different kinases have differential preferences for predicted disordered phosphoacceptors and

133 found that PknA has a slightly higher preference for disordered phosphoacceptors compared with

134 other kinases (Supplemental Figure 2). However, the uncorrected Chi-square test p-value was

135 only 0.04 , whereas other kinases showed uncorrected $\mathrm{p}$-values $>0.05$.

136 Mass-spectrometry has been used to identify S/T phosphorylation sites in a number of

137 prokaryotes other than M. tuberculosis (Mijakovic and Macek, 2012). Finally, I tested the

138 association between disorder and phosphorylation in other prokaryotes, and found that an

139 association between disorder and phosphorylation was present in some prokaryotes but not in

140 others (Table 1).

\section{Discussion}

142 Here, I show the enrichment of localized S/T phosphosites in disordered regions of proteins in $M$.

143 tuberculosis. $\mathrm{pS} / \mathrm{T}$ sites in M. tuberculosis are approximately 2 fold more likely to occur in

144 disordered regions compared with ordered regions (Figure 1). This preference is similar to that

145 observed in eukaryotes, where $\mathrm{pS} / \mathrm{T}$ sites are 2-to-3 fold more likely to occur in disordered

146 regions (Amoutzias et al., 2012; Landry et al., 2009; Marchini et al., 2011). However, because the 
147 percentage of disordered residues is much higher in eukaryotes than in M. tuberculosis,

148 approximately $80-90 \%$ of $\mathrm{pS} / \mathrm{T}$ sites in eukaryotes occur in disordered regions (Amoutzias et al.,

149 2012; Collins et al., 2008; Landry et al., 2009; Marchini et al., 2011) compared to approximately

$15040 \%$ in M. tuberculosis (Figure 1). The association between protein disorder and phosphorylation

151 may offer similar advantages as those proposed in eukaryotes, including binding to multiple

152 partners and transient mode of interaction (Dunker et al., 2005; Dyson and Wright, 2005; Fink,

153 2005; Iakoucheva et al., 2004; Patil and Nakamura, 2006; Singh et al., 2007; Tompa, 2002;

154 Tompa et al., 2005; Wright and Dyson, 1999), which are a prerequisite for regulatory interactions.

155 Thus M. tuberculosis and eukaryotic S/T phosphorylation dependent regulation may be more

156 similar than generally appreciated. Whereas most bacteria have a low amount of protein disorder,

157 M. tuberculosis has a high disorder content. The high disorder content in M. tuberculosis may

158 possibly allow higher levels of S/T phosphorylation. Some other bacteria with high disorder

159 content (Streptomyces coelicolor and Halobacterium salinarum) also show an association

160 between disorder and $\mathrm{S} / \mathrm{T}$ phosphorylation, whereas bacteria with low disorder contents do not

161 (Table 1). The exception is Thermus thermophilus, which exhibited an enrichment of

162 phosphorylation in disordered regions with marginal statistical significance (Table 1). Previously,

163 a large fraction of phosphosites in Thermus thermophilus was observed in loop regions (Takahata

164 et al., 2012), though no statistical test for enrichment was performed. It would be interesting to

165 study S/T phosphorylation in other high disorder-containing bacteria to test whether these

166 bacteria also show high levels of S/T phosphorylation.

167 PknA has a slightly higher preference for disordered phosphoacceptor sites on synthetic 13-mer

168 substrate peptides under in vitro conditions than other kinases (Supplemental Figure 2). However,

169 these results should be taken with the caveat that the structure of a peptide under in vitro

170 conditions might be very different from the in vivo structure in the context of the full protein. 


\section{PeerJ Reviewing Manuscript}

171 Thus the differential preferences of kinases towards disordered substrates under in vivo

172 conditions remains an open question.

173 In eukaryotes, the conservation of $\mathrm{pS} / \mathrm{T}$ sites has been a matter of some debate, with some studies

174 reporting no higher conservation of $\mathrm{pS} / \mathrm{T}$ sites (Gnad et al., 2009; Landry et al., 2009), while

175 others reported higher conservation of pS/T sites (Chen et al., 2010; Gray and Kumar, 2011).

176 More recently, it was argued that pooling conservation rates from multiple proteins may bias the

177 results since this approach does not account for the large differences in the conservation of

178 different proteins. Thus, the conservation of $\mathrm{pS} / \mathrm{T}$ sites should be compared with the conservation

179 of npS/T sites from the same protein (Gray and Kumar, 2011). Indeed, in M. tuberculosis, there

180 was no significant difference in the conservation of $\mathrm{pS} / \mathrm{T}$ and $\mathrm{npS} / \mathrm{T}$ sites among mycobacteria,

181 when their averages were compared across proteins (mean conservation in 11.34 and 11.28

182 species out of 14 , respectively; Wilcox test $p$ 0.4). However, when the conservation of $\mathrm{pS} / \mathrm{T}$ was

183 compared with that of $\mathrm{npS} / \mathrm{T}$ from the same protein, $\mathrm{pS} / \mathrm{T}$ sites were found to be more conserved

184 relative to $\mathrm{npS} / \mathrm{T}$ sites. It might be useful to prioritize $\mathrm{pS} / \mathrm{T}$ sites with high conservation (relative

185 to $\mathrm{npS} / \mathrm{T}$ sites from the same protein) for further experimental studies. Phosphoproteomic

186 analyses on more mycobacteria would also be highly valuable to identify S/T sites

187 phosphorylated in multiple mycobacteria. In eukaryotes, disordered $\mathrm{pS} / \mathrm{T}$ sites demonstrate

188 higher rate of evolution (Landry et al., 2009). In M. tuberculosis, I did not find statistically

189 significant difference in the conservation of disordered $\mathrm{pS} / \mathrm{T}$ sites, compared with disordered

$190 \mathrm{npS} / \mathrm{T}$ sites. However, due to the low number of sites and proteins analyzed, this issue would

191 need to be revisited when more data become available.

192 Further important questions for the future include the following, 1) Are disordered and ordered 193 S/T phosphosites functionally different? and 2) Do different kinases differ in their preferences 
194 for disorder in their substrates under physiological conditions? Incorporating disorder

195 information might also be useful for the prediction of novel S/T phosphosites (Miller et al.,

196 2009), as has been shown in eukaryotes (Iakoucheva et al., 2004; Neduva et al., 2005). S/T

197 kinases and their substrates have been linked to the survival, pathogenesis and virulence of $M$.

198 tuberculosis (Av-Gay and Everett, 2000; Cousin et al., 2013; Pereira et al., 2011). Thus, these

199 finding may facilitate an understanding of the basic biology of this deadly pathogen.

200 Acknowledgements

201 I would like to thank Dr. Shampa Ghosh from Bionivid Technology, and Dr. Rahul Modak and

202 Dr. Avinash Sonawane from KIIT University for critical reading of the manuscript.

References

204 1. Aivaliotis M, Macek B, Gnad F, Reichelt P, Mann M and Oesterhelt D . 2009. Ser/Thr/Tyr protein 205 phosphorylation in the archaeon Halobacterium salinarum--a representative of the third domain of $206 \quad$ life. PLoS One 4: e4777.

207 2. Albuquerque CP, Smolka MB, Payne SH, Bafna V, Eng J and Zhou H . 2008. A multidimensional 208 chromatography technology for in-depth phosphoproteome analysis. Mol Cell Proteomics 7:

$209 \quad 1389-1396$.

210 3. Amoutzias GD, He Y, Lilley KS, Van de PY and Oliver SG . 2012. Evaluation and properties of 211 the budding yeast phosphoproteome. Mol Cell Proteomics 11: M111.

212 4. Av-Gay Y and Everett M . 2000. The eukaryotic-like Ser/Thr protein kinases of Mycobacterium 213 tuberculosis. Trends Microbiol 8: 238-244. 
214 5. Brown CJ, Takayama S, Campen AM, Vise P, Marshall TW, Oldfield CJ, Williams CJ and Dunker

215 AK . 2002. Evolutionary rate heterogeneity in proteins with long disordered regions. J Mol Evol

$216 \quad 55: 104-110$.

217 6. Chen SC, Chen FC and Li WH . 2010. Phosphorylated and nonphosphorylated serine and

218 threonine residues evolve at different rates in mammals. Mol Biol Evol 27: 2548-2554.

219 7. Collins MO, Yu L, Campuzano I, Grant SG and Choudhary JS . 2008. Phosphoproteomic analysis

220 of the mouse brain cytosol reveals a predominance of protein phosphorylation in regions of

221 intrinsic sequence disorder. Mol Cell Proteomics 7: 1331-1348.

222 8. Combet C, Blanchet C, Geourjon C and Deleage G. 2000. NPS@: network protein sequence 223 analysis. Trends Biochem Sci 25: 147-150.

224 9. Cousin C, Derouiche A, Shi L, Pagot Y, Poncet S and Mijakovic I . 2013. Protein-

225 serine/threonine/tyrosine kinases in bacterial signaling and regulation. FEMS Microbiol Lett 346:

$226 \quad 11-19$

227 10. Deleage G, Blanchet C and Geourjon C . 1997. Protein structure prediction. Implications for the 228 biologist. Biochimie 79: 681-686.

229 11. Dosztanyi Z, Csizmok V, Tompa P and Simon I . 2005a. IUPred: web server for the prediction of 230 intrinsically unstructured regions of proteins based on estimated energy content. Bioinformatics $231: 3433-3434$

232 12. Dosztanyi Z, Csizmok V, Tompa P and Simon I . 2005b. The pairwise energy content estimated 233 from amino acid composition discriminates between folded and intrinsically unstructured proteins. $234 \quad$ J Mol Biol 347: 827-839.

235 13. Dunker AK, Cortese MS, Romero P, Iakoucheva LM and Uversky VN . 2005. Flexible nets. The 236 roles of intrinsic disorder in protein interaction networks. FEBS J 272: 5129-5148. 
237 14. Dyson HJ and Wright PE . 2005. Intrinsically unstructured proteins and their functions. Nat Rev $238 \quad$ Mol Cell Biol 6: 197-208.

239 15. Fink AL . 2005. Natively unfolded proteins. Curr Opin Struct Biol 15: 35-41.

240 16. Gnad F, de Godoy LM, Cox J, Neuhauser N, Ren S, Olsen JV and Mann M . 2009. High-accuracy

241 identification and bioinformatic analysis of in vivo protein phosphorylation sites in yeast.

$242 \quad$ Proteomics 9: 4642-4652.

243 17. Gray VE and Kumar S . 2011. Rampant purifying selection conserves positions with posttranslational modifications in human proteins. Mol Biol Evol 28: 1565-1568.

245 18. Gsponer J, Futschik ME, Teichmann SA and Babu MM . 2008. Tight regulation of unstructured proteins: from transcript synthesis to protein degradation. Science 322: 1365-1368.

247 19. Iakoucheva LM, Radivojac P, Brown CJ, O'Connor TR, Sikes JG, Obradovic Z and Dunker AK . 248 2004. The importance of intrinsic disorder for protein phosphorylation. Nucleic Acids Res 32:

$249 \quad 1037-1049$.

250 20. Kobir A, Shi L, Boskovic A, Grangeasse C, Franjevic D and Mijakovic I . 2011. Protein 251 phosphorylation in bacterial signal transduction. Biochim Biophys Acta 1810: 989-994.

252 21. Landry CR, Levy ED and Michnick SW . 2009. Weak functional constraints on 253 phosphoproteomes. Trends Genet 25: 193-197.

254 22. Lew JM, Mao C, Shukla M, Warren A, Will R, Kuznetsov D, Xenarios I, Robertson BD, Gordon 255 SV, Schnappinger D, Cole ST and Sobral B . 2013. Database resources for the tuberculosis 256 community. Tuberculosis (Edinb ) 93: 12-17.

257 23. Lin MH, Hsu TL, Lin SY, Pan YJ, Jan JT, Wang JT, Khoo KH and Wu SH . 2009.

258 Phosphoproteomics of Klebsiella pneumoniae NTUH-K2044 reveals a tight link between tyrosine 259 phosphorylation and virulence. Mol Cell Proteomics 8: 2613-2623. 
260

261

262

263

264

265

266

267

268

269

270

271

272

273

274

275

276

277

278

279

280

281

24. Macek B, Mijakovic I, Olsen JV, Gnad F, Kumar C, Jensen PR and Mann M . 2007. The serine/threonine/tyrosine phosphoproteome of the model bacterium Bacillus subtilis. Mol Cell Proteomics 6: 697-707.

25. Manteca A, Ye J, Sanchez J and Jensen ON . 2011. Phosphoproteome analysis of Streptomyces development reveals extensive protein phosphorylation accompanying bacterial differentiation. J Proteome Res 10: 5481-5492.

26. Marchini FK, de Godoy LM, Rampazzo RC, Pavoni DP, Probst CM, Gnad F, Mann M and Krieger MA . 2011. Profiling the Trypanosoma cruzi phosphoproteome. PLoS One 6: e25381.

27. Mijakovic I and Macek B . 2012. Impact of phosphoproteomics on studies of bacterial physiology. FEMS Microbiol Rev 36: 877-892.

28. Miller ML, Soufi B, Jers C, Blom N, Macek B and Mijakovic I . 2009. NetPhosBac - a predictor for Ser/Thr phosphorylation sites in bacterial proteins. Proteomics 9: 116-125.

29. Misra SK, Milohanic E, Ake F, Mijakovic I, Deutscher J, Monnet V and Henry C . 2011. Analysis of the serine/threonine/tyrosine phosphoproteome of the pathogenic bacterium Listeria monocytogenes reveals phosphorylated proteins related to virulence. Proteomics 11: 4155-4165.

30. Mizianty MJ, Peng ZL and Kurgan L . 2013. MFDp2 - accurate predictor of disorder in proteins by fusion of disorder probabilities, content and profiles.

31. Mizianty MJ, Uversky V and Kurgan L . 2014. Prediction of intrinsic disorder in proteins using MFDp2. Methods Mol Biol 1137:147-62. doi: 10.1007/978-1-4939-0366-5_11.: 147-162.

32. Monastyrskyy B, Kryshtafovych A, Moult J, Tramontano A and Fidelis K . 2014. Assessment of protein disorder region predictions in CASP10. Proteins 82 Suppl 2:127-37. doi: 10.1002/prot.24391. Epub;\%2013 Nov 22.: 127-137. 
282

283

284

285

286

287

288

289

290

291

292

293

294

295

296

297

298

299

300

301

302

303

33. Neduva V, Linding R, Su-Angrand I, Stark A, de MF, Gibson TJ, Lewis J, Serrano L and Russell RB . 2005. Systematic discovery of new recognition peptides mediating protein interaction networks. PLoS Biol 3: e405.

34. Parker JL, Jones AM, Serazetdinova L, Saalbach G, Bibb MJ and Naldrett MJ . 2010. Analysis of the phosphoproteome of the multicellular bacterium Streptomyces coelicolor A3(2) by protein/peptide fractionation, phosphopeptide enrichment and high-accuracy mass spectrometry. Proteomics 10: 2486-2497.

35. Patil A and Nakamura H . 2006. Disordered domains and high surface charge confer hubs with the ability to interact with multiple proteins in interaction networks. FEBS Lett 580: 2041-2045.

36. Pereira SF, Goss L and Dworkin J . 2011. Eukaryote-like serine/threonine kinases and phosphatases in bacteria. Microbiol Mol Biol Rev 75: 192-212.

37. Prisic S, Dankwa S, Schwartz D, Chou MF, Locasale JW, Kang CM, Bemis G, Church GM, Steen H and Husson RN . 2010. Extensive phosphorylation with overlapping specificity by Mycobacterium tuberculosis serine/threonine protein kinases. Proc Natl Acad Sci U S A \%20;107: $7521-7526$.

38. Sievers F and Higgins DG . 2014. Clustal Omega, accurate alignment of very large numbers of sequences. Methods Mol Biol 1079:105-16. doi: 10.1007/978-1-62703-646-7_6.: 105-116.

39. Singh GP, Ganapathi M and Dash D . 2007. Role of intrinsic disorder in transient interactions of hub proteins. Proteins 66: 761-765.

40. Soufi B, Gnad F, Jensen PR, Petranovic D, Mann M, Mijakovic I and Macek B . 2008. The Ser/Thr/Tyr phosphoproteome of Lactococcus lactis IL1403 reveals multiply phosphorylated proteins. Proteomics 8: 3486-3493. 
304 41. Takahata Y, Inoue M, Kim K, Iio Y, Miyamoto M, Masui R, Ishihama Y and Kuramitsu S . 2012.

305 Close proximity of phosphorylation sites to ligand in the phosphoproteome of the extreme

306 thermophile Thermus thermophilus HB8. Proteomics 12: 1414-1430.

307 42. Tompa P. 2002. Intrinsically unstructured proteins. Trends Biochem Sci 27: 527-533.

308 43. Tompa P, Szasz C and Buday L . 2005. Structural disorder throws new light on moonlighting.

309 Trends Biochem Sci 30: 484-489.

310 44. Uversky VN, Gillespie JR and Fink AL . 2000. Why are "natively unfolded" proteins unstructured $311 \quad$ under physiologic conditions? Proteins 41: 415-427.

312 45. Walsh I, Martin AJ, Di DT and Tosatto SC . 2012. ESpritz: accurate and fast prediction of protein 313 disorder. Bioinformatics 28: 503-509.

314 46. Ward JJ, Sodhi JS, McGuffin LJ, Buxton BF and Jones DT . 2004. Prediction and functional 315 analysis of native disorder in proteins from the three kingdoms of life. J Mol Biol 337: 635-645.

316 47. Wolf YI and Koonin EV . 2012. A tight link between orthologs and bidirectional best hits in 317 bacterial and archaeal genomes. Genome Biol Evol 4: 1286-1294.

318 48. Wright PE and Dyson HJ . 1999. Intrinsically unstructured proteins: re-assessing the protein 319 structure-function paradigm. J Mol Biol 293: 321-331.

320 49. Yang MK, Qiao ZX, Zhang WY, Xiong Q, Zhang J, Li T, Ge F and Zhao JD . 2013. Global 321 phosphoproteomic analysis reveals diverse functions of serine/threonine/tyrosine phosphorylation 322 in the model cyanobacterium Synechococcus sp. strain PCC 7002. J Proteome Res 12: 1909-1923. 


\section{Figure Legends}

324 Figure 1. Phosphorylated serine/threonine sites in $\mathbf{M}$. tuberculosis are more likely to be

325 disordered. Both phosphorylated and non-phosphorylated serine/threonine sites are from

326 the same set of proteins. The disorder was predicted using the IUPred, ESpritz and MFDp2

327 methods. The Fisher test $p$ values were $6 \mathrm{e}-8,8 \mathrm{e}-14$ and $6 \mathrm{e}-15$, respectively. $\mathrm{pS} / \mathrm{T}$

328 -phosphorylated serine/threonine, $\mathrm{npS} / \mathrm{T}$ - non-phosphorylated serine/threonine. Error bars

329 indicate $95 \%$ confidence intervals of the mean from 1000 bootstrap samples.

330 Figure 2. Association between predicted secondary structure and phosphorylation of

331 serine/threonine sites in M. tuberculosis. Phosphorylated serine/threonine sites occur

332 preferentially in coil regions, are significantly depleted in sheet regions and show no

333 significant difference in helix regions. The Fisher test $p$ values are 2e-5, 4e-4 and 0.2,

334 respectively. pS/T -phosphorylated serine/threonine, npS/T- non-phosphorylated

335 serine/threonine. Error bars indicate $95 \%$ confidence intervals of the mean from 1000

336 bootstrap samples. 


\section{1}

Phosphorylated serine/threonine sites in M. tuberculosis are more likely to be disordered

Figure 1. Phosphorylated serine/threonine sites in M. tuberculosis are more likely to be disordered. Both phosphorylated and non-phosphorylated serine/threonine sites are from the same set of proteins. The disorder was predicted using the IUPred, ESpritz and MFDp2 methods. The Fisher test $p$ values were $6 \mathrm{e}-8,8 \mathrm{e}-14$ and $6 \mathrm{e}-15$, respectively. pS/T phosphorylated serine/threonine, npS/T- non-phosphorylated serine/threonine. Error bars indicate $95 \%$ confidence intervals of the mean from 1000 bootstrap samples.

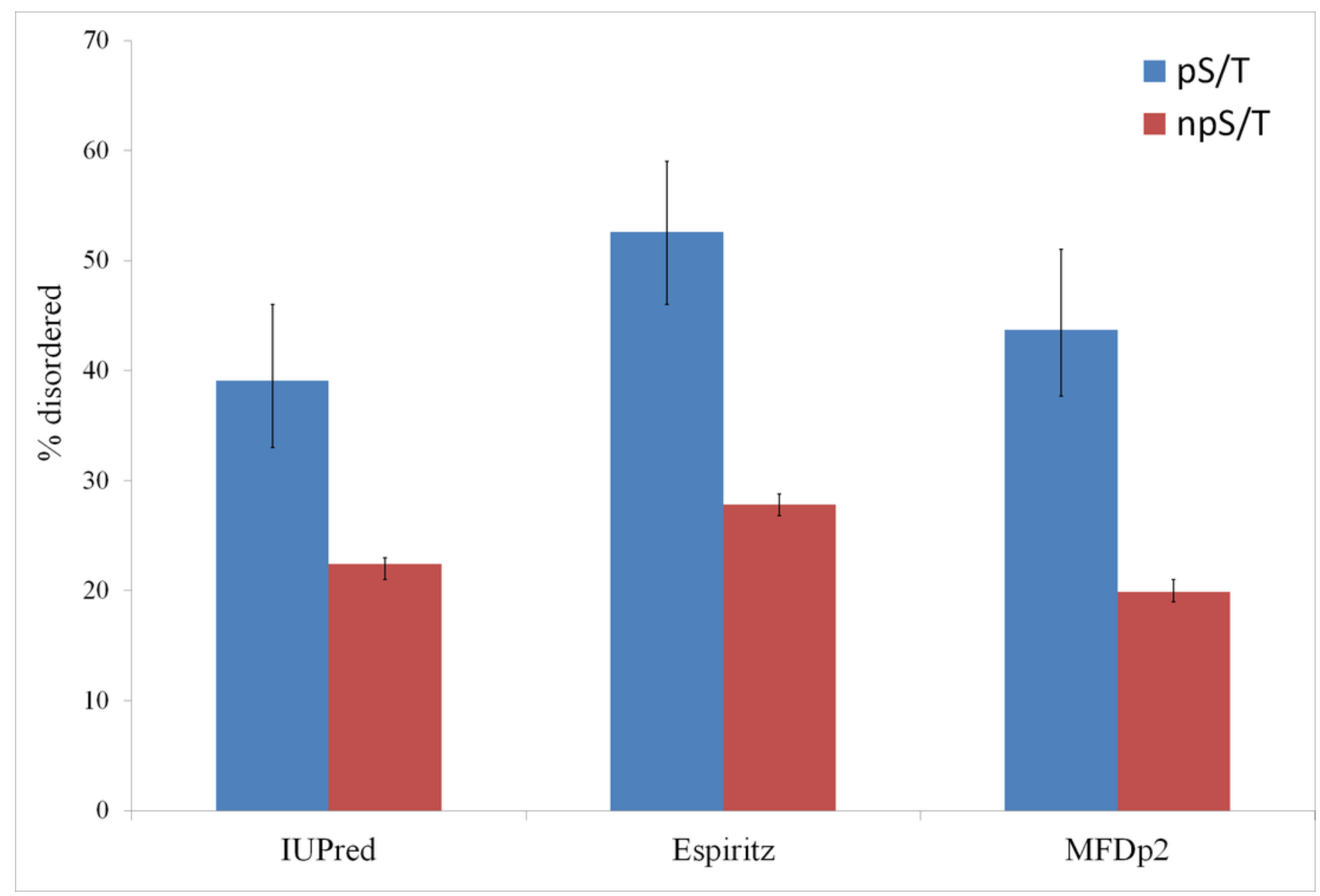


2

Association between predicted secondary structure and phosphorylation of serine/threonine sites in $M$. tuberculosis

Figure 2. Association between predicted secondary structure and phosphorylation of serine/threonine sites in $\mathbf{M}$. tuberculosis. Phosphorylated serine/threonine sites occur preferentially in coil regions, are significantly depleted in sheet regions and show no significant difference in helix regions. The Fisher test $p$ values are $2 \mathrm{e}-5,4 \mathrm{e}-4$ and 0.2 , respectively. $\mathrm{pS} / \mathrm{T}$-phosphorylated serine/threonine, $\mathrm{npS} / \mathrm{T}$ - non-phosphorylated serine/threonine. Error bars indicate $95 \%$ confidence intervals of the mean from 1000 bootstrap samples.

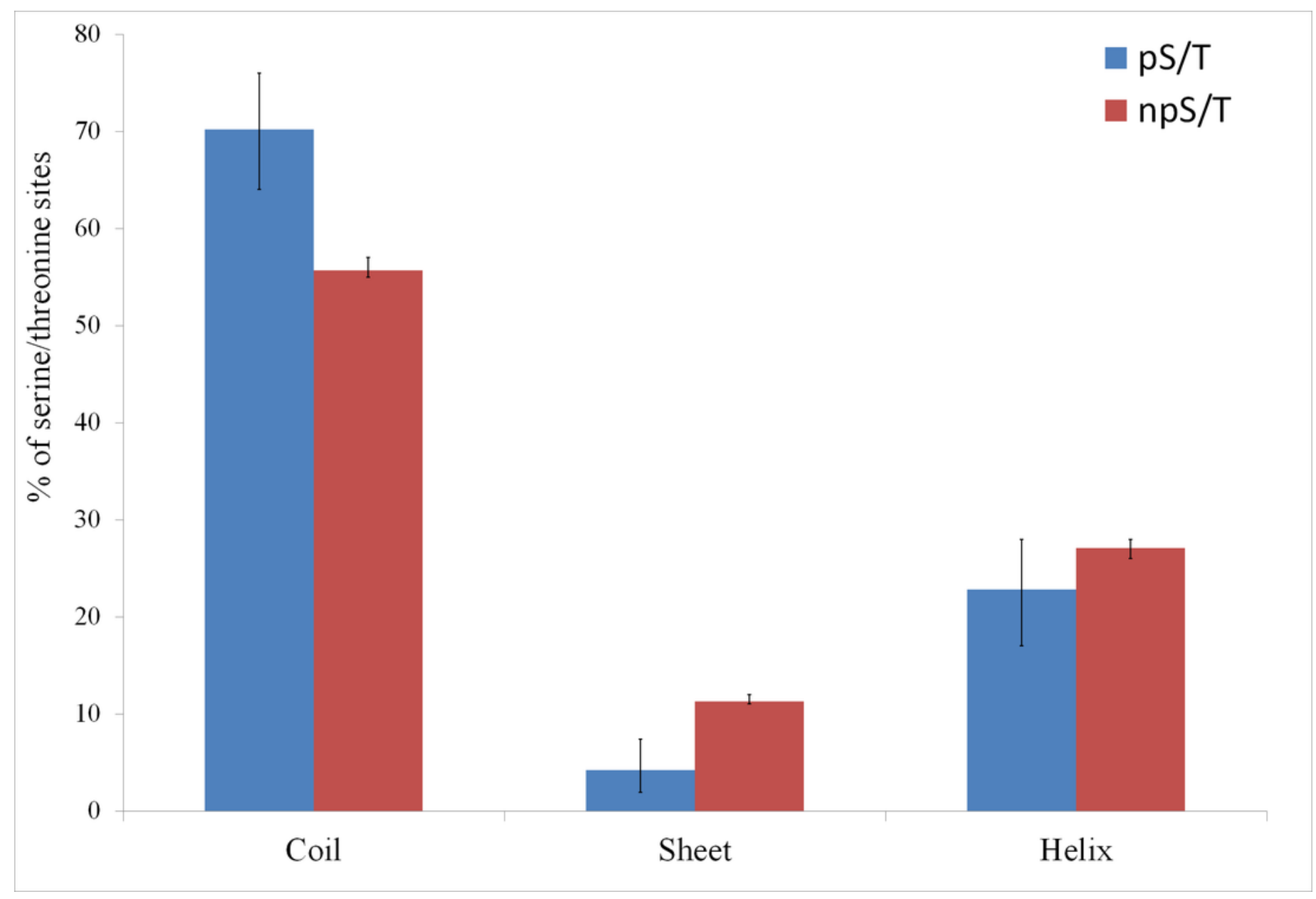




\section{Table $\mathbf{1}$ (on next page)}

Number of disordered and ordered serine/threonine sites in phosphoproteomes of different bacteria 
Table 1. Number of disordered and ordered serine/threonine sites in phosphoproteomes of different bacteria".

Organism

Mycobacterium tuberculosis

Escherichia coli

Bacillus subtilis

Thermus thermophilus

Streptomyces coelicolor

Streptomyces coelicolor*

Klebsiella pneumoniae

Lactobacillus lactis

Synechococcus sp.

Halobacterium salinarum

Listeria monocytogenes

Mycoplasma pneumoniae

Streptococcus pneumoniae

Number of
localized pST
21
9
9
4
2
21
44
66
354
75
65
14

* S. coelicolor appears twice because of two independent studies on S/T phosphorylation \# disorder predicted using the IUPred

method

\begin{tabular}{|c|c|c|c|}
\hline $\begin{array}{l}\text { \% pST } \\
\text { disordered }\end{array}$ & $\begin{array}{l}\text { \% npST } \\
\text { disordered }\end{array}$ & $\begin{array}{l}\text { Fisher } \\
\text { test p- } \\
\text { value }\end{array}$ & $\begin{array}{l}\% \\
\text { proteome } \\
\text { disordered }\end{array}$ \\
\hline 39.1 & 22.4 & $6 \mathrm{E}-08$ & 11.7 \\
\hline 8.2 & 5.3 & 0.25 & 5.2 \\
\hline 4.3 & 8.7 & 0.18 & 5.7 \\
\hline 11.9 & 4.2 & 0.04 & 4.9 \\
\hline 60.0 & 36.8 & 0.06 & 18.6 \\
\hline 64.5 & 40.0 & $3 \mathrm{E}-12$ & 18.6 \\
\hline 11.4 & 9.9 & 0.80 & 4.9 \\
\hline 18.2 & 15.3 & 0.49 & 5.0 \\
\hline 10.7 & 12.9 & 0.26 & 6.8 \\
\hline 64.4 & 38.7 & $2 \mathrm{E}-05$ & 20.9 \\
\hline 11.7 & 13.5 & 0.68 & 5.0 \\
\hline 9.2 & 16.4 & 0.16 & 9.0 \\
\hline 12.2 & 14.0 & 0.63 & 5.0 \\
\hline
\end{tabular}

